\title{
Update on HIV integrase inhibitors for the treatment of HIV-1 infection
}

\author{
Blake Max ${ }^{*, 1,2}$ \\ ${ }^{1}$ Clinical Associate Professor, College of Pharmacy, University of Illinois at Chicago, IL 60612-7229, USA \\ ${ }^{2}$ HIV Clinical Pharmacist Ruth M Rothstein CORE Center, Cook County Health \& Hospitals System, IL 60612, USA \\ *Author for correspondence: bmax@cookcountyhhs.org
}

It has been over 30 years since the first antiretroviral agent was approved for treatment of HIV-1 infection and its impact on morbidity and mortality has been dramatic. However, early treatments were hindered by short- and long-term toxicity, poor tolerability, high pill burden, drug interactions and development of drug resistance. A major breakthrough in HIV therapeutics occurred over a decade ago with a new class of drugs that not only are preferred by HIV treatment guidelines but also are changing the HIV treatment paradigm. This new class of drugs are called HIV-1 integrase strand transfer inhibitors and they have established a role in almost every aspect of HIV treatment.

First draft submitted: 11 July 2019; Accepted for publication: 7 October 2019; Published online: 17 December 2019

Keywords: AIDS • antiretroviral therapy • bictegravir • cabotegravir • dolutegravir • elvitegravir • HIV • INSTI • integrase strand transfer inhibitors • raltegravir

It has been over a decade since integrase strand transfer inhibitors (INSTIs) have made their impact on the treatment of HIV infection. Raltegavir (Isentress ${ }^{\circledR}$ ) was the first INSTI US FDA approved (October 2007) and became a preferred agent for treatment naive HIV-1-infected adults recommended by the Department of Health and Human Services (DHHS) guidelines in 2009. Since the addition of raltegravir to the HIV treatment armamentarium, there have been three additional INSTIs FDA approved (elvitegravir, dolutegravir, bictegravir) and a fourth (cabotegravir) in advanced clinical trial development. In 2019, the DHHS adult and adolescent treatment guidelines recommend raltegravir, dolutegravir or bictegravir as the preferred anchor in combination with two nucleoside reverse transcriptase inhibitors (NRTIs) for antiretroviral treatment naive patients [1]. This recommendation is based on durable virologic suppression, excellent tolerability, minimal toxicity and ease of use. INSTIs have established a critical role in almost every aspect of HIV treatment including treatment naive and experienced patients, adolescent, pediatric, pregnancy and postexposure prophylaxis. Emerging data for preexposure prophylaxis (PrEP) also appear to be a future role and finally changing the HIV treatment paradigm from three-drug therapy to two-drug therapy was recently validated with dolutegravir. However, like all antiretroviral agents, INSTIs are not without clinical sequelae that have tempered some of the enthusiasm. This article will review important clinical data and place in therapy for this indispensable class of medications for HIV-1 treatment.

\section{Mechanism of action}

INSTIs are one of seven classes of antiretroviral agents used to treat HIV-1 infection. HIV-1 integrase is a viralspecific enzyme that is essential for viral replication and therefore makes an excellent target for antiretroviral therapy (ART). The insertion of viral DNA into the host chromosome is carried out by HIV-1 integrase. This multistep process starts when double-stranded viral DNA undergoes 3 '-endonucleolytic processing in the cytoplasm and is subsequently carried into the cell nucleus by HIV-1 integrase as the preintegration complex [2-4]. This enzyme has two distinct catalytic reactions: in the cytoplasm, $3^{\prime}$-end processing occurs where a pair of dinucleotides are removed at the $3^{\prime}$-end of the long terminal repeat on each proviral strand. The second step, also known as strand transfer, occurs in the nucleus where HIV-1 integrase nonspecifically cuts cellular DNA and the two viral 3 '-ends are inserted into host DNA. The integration process is completed after covalent ligation of the inserted proviral DNA and cellular enzymes repair single-strand gaps between host and viral DNA. The mechanism of action for 

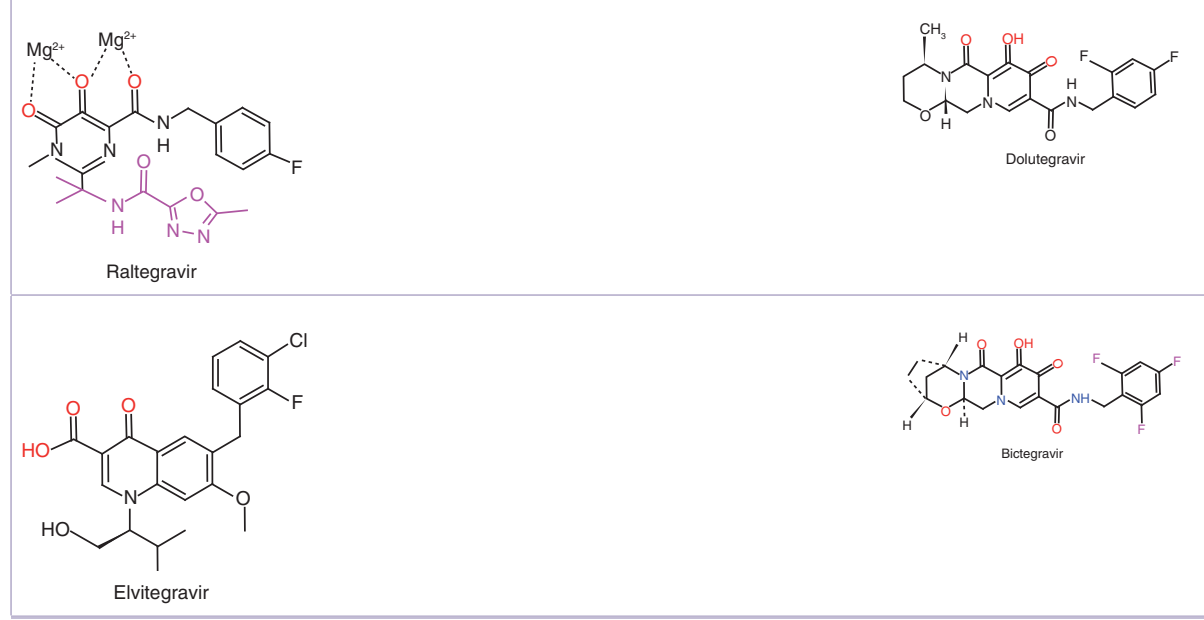

INSTIs occurs during the second step (strand transfer within the nucleus). The catalytic domain in HIV-1 integrase is highly conserved and requires divalent cations $(\mathrm{Mg}[2+]$ or $\mathrm{Mn}[2+])$ in coordination with a triad of carboxylate amino acid residues to carry out the strand transfer step [5]. INSTIs inhibit HIV-1 integrase by chelation of divalent metal cations in the enzyme catalytic site [2]. All INSTIs have a structural motif, diketo acid moiety (Table 1) and a hydrophobic region that allows for binding within the space created by integrase and the $3^{\prime}$-HIV-1 DNA dinucleotide end.

\section{Raltegravir}

Raltegravir was the first of this class of antiretrovirals to be used in clinical practice. ART in the 1990s and early 2000s targeted HIV reverse transcriptase and HIV protease enzymes. However, these drugs were limited by the ability of the virus to develop drug specific mutations rendering them less effective and capable of transmitting drug resistant virus. The discovery of HIV integrase as a new target for therapeutic intervention came at a critical time when loss of drug effectiveness due to the development of drug resistance, cross resistance and multidrug resistance was impacting clinical outcomes. The high potency of raltegravir, a characteristic of all INSTIs, was observed in a 10-day monotherapy trial that showed a mean $2.0 \log _{10}$ copies/ml reduction in HIV viral load [6]. The BENCHMRK-1 and 2 trials were critical in establishing antiviral efficacy in patients who had resistance to multiple antiretroviral agents. These two large Phase III trials compared patients randomized to receive raltegravir or placebo plus optimized background regimen $[7,8]$. The results from these two trials demonstrated that raltegravir was superior to placebo and thus laid the foundation for combining raltegravir with other active drugs, preferably 2-3 active drugs to achieve optimal viral suppression in treatment-experienced patients.

After the promising results from the BENCHMRK studies, raltegravir was compared with the single tablet regimen efavirenz/emtricitabine/tenofovir disoproxil (Atripla ${ }^{\circledR}$ ) in treatment-naive patients, the gold standard at the time. The 48-week results from the STRTMRK trial showed that raltegravir + emtricitabine/tenofovir disoproxil (Truvada ${ }^{\circledR}$ ) was noninferior to Atripla [9]. Another finding from this study was those patients who received raltegravir achieved virologic suppression in a shorter time (weeks 2-16) compared with Atripla. This pharmacodynamic characteristic is also observed for other INSTIs; however, clinical benefit from a more rapid decline in viral load has not been established. Raltegravir also demonstrated a favorable safety and tolerability profile compared with efavirenz, was equally efficacious across all viral load and CD4 T-lymphocyte stratifications, and had minimal impact on serum lipids. There were few virologic failures in patients receiving raltegravir, however, drug resistance with known integrase mutations were documented in half the patients who had virologic failure. Despite all the favorable characteristics that raltegravir possessed, the acquisition of drug resistance would be a driving force in the development of 'second-generation' INSTIs.

Switch studies have become a standard clinical trial design for INSTIs. The concept of switch studies is predicated on reducing side effects of older antiretrovirals, improve lipid abnormalities or other metabolic complications associated with antiretrovirals, preventing/avoiding drug interactions and reduce pill burden or regimen simpli- 
fication [10]. The SWITCHMRK study compared patients who had a HIV viral load $\leq 50$ copies $/ \mathrm{ml}$ for at least 3 months on a protease inhibitor (PI)-based regimen and were randomized to remain on the same PI or switch to raltegravir [11]. In this study, patients were prescribed lopinavir/ritonavir (Kaletra ${ }^{\circledR}$ ) and not surprisingly those patients who switched to raltegravir had statistically greater reductions in lipids, while other clinical and laboratory adverse events occurred at similar frequencies. However, this study was terminated at week 24 because of lower than expected virologic efficacy in the raltegravir switch group compared with those patients who remained on Kaletra. Further analysis revealed that patients who had previous virologic failure maintained a higher rate of virologic suppression in the Kaletra arm compared with patients who switched to raltegravir. There was no difference in viral suppression between treatment groups in patients without previous virologic failure. This observation showed the vulnerability of raltegravir, which has a low genetic barrier to resistance if it is not paired with other active drugs.

One of the advantages of raltegravir is that is has been studied in many patient populations. Unlike other INSTIs, raltegravir can be used in children starting at birth to age 3 who weigh $\geq 2 \mathrm{~kg}$ [12]. Raltegravir $400 \mathrm{mg}$ twice daily is also recommended as one of the preferred agents for all patients more than 4 weeks of age for both nonoccupational and occupational HIV exposure prophylaxis [13,14]. Pharmacokinetics, safety and efficacy have also been studied in pregnant women and results showed raltegravir is well tolerated, safe and does not require dose adjustment [15,16]. Raltegravir has an excellent safety record with few discontinuations due to adverse effects in clinical trials; however, elevated creatinine kinase has been observed with a few cases of rhabdomyolysis reported in the literature [17].

Raltegravir ushered in a new class of antiretrovirals and possesses many characteristics that made it a key contributor to improved clinical outcomes for HIV-1-infected patients. However, despite the benefits raltegravir use in clinical practice has waned with the development of second-generation INSTIs. The disadvantages of raltegravir are that it is primarily dosed one tablet $(400 \mathrm{mg})$ twice daily, although there is an FDA-approved 600-mg tablet that is dosed two tablets once daily. Raltegravir is also not coformulated as a single tablet regimen, which remains the gold standard for HIV treatment to reduce pill burden. Most importantly, raltegravir has a low genetic barrier to resistance; therefore, if a patient fails raltegravir-based regimen, they will likely develop integrase drug mutations that can confer cross resistance to other drugs in the class [18].

\section{Elvitegravir}

ART has undergone significant improvement over the years, one of the most important has been the development of once daily single tablet regimens. In 2012, elvitegravir became the second INSTI FDA approved. Elvitegravir was initially available as a single tablet regimen coformulated with cobicistat, emtricitabine and tenofovir disoproxil (Stribild ${ }^{\circledR}$ ). Unlike other INSTIs, elvitegravir is metabolized by CYP450 3A4. The addition of cobicistat, a CYP450 3A4 inhibitor that has no antiviral activity, increases elvitegravir plasma levels permitting once daily dosing [19]. However, this pharmacokinetic benefit comes with a price of numerous potential drug interactions with coadministered medications metabolized by CYP450 3A4. Cobicistat also effects renal secretion of creatinine by reversible inhibition of renal transporters OAT2 and MATE-1 [20]. This can result in small increases in plasma serum creatinine with a corresponding decrease in estimated glomerular filtration rate (eGFR) approximately 10$14 \mathrm{ml} / \mathrm{min}$ [21]. Changes in both laboratory markers (serum creatinine and eGFR) are nonpathogenic, can occur within days of starting cobicistat, return to baseline when discontinued, not indicative of worsening renal function and do not warrant drug discontinuation.

Despite favorable clinical trials, the utility of Stribild was relatively short and gave way to a new and improved single tablet-coformulated regimen elvitegravir/cobicistat/emtricitabine/tenofovir alafenamide (Genvoya ${ }^{\circledR}$ ) that was FDA approved in 2015. Genvoya was able to capitalize on combining elvitegravir with an improved NRTI tenofovir alafenamide that has less renal and bone toxicity than tenofovir disoproxil [22,23]. Tenofovir alafenamide has shown improved safety as well as more potent antiviral activity compared with tenofovir disoproxil [24,25]. With the improved formulation of tenofovir, Genvoya was compared with Stribild in two controlled, double-blind, Phase III studies. Pooled results through 48 weeks showed that Genvoya was noninferior to Stribild and both formulations were well tolerated with few discontinuations [26]. Observations from these clinical trials showed that patients who received Genvoya had smaller reductions in eGFR, more favorable changes in specific markers of proximal renal tubular dysfunction and more favorable effect on hip and spine bone mineral density when compared with patients on Stribild. However, small increases in triglycerides, total cholesterol and low-density lipoproteins were observed in patients on Genvoya compared with Stribild. More importantly, although virologic failure was uncommon when it did occur the majority of patients developed primary INSTI drug mutations. 
INSTIs have favorable pharmacokinetic and pharmacodynamic characteristics that make them excellent candidates for switch studies in virologically suppressed patients. Researchers learned from the SWITCHMRK study that switching patients from a drug with a high genetic barrier to resistance (Kaletra) to one with a lower genetic barrier (raltegravir) can result in virologic failure. Elvitegravir has been evaluated in two randomized, controlled switch studies designed for patients with no previous virologic failure, no resistance to emtricitabine or tenofovir, and undetectable HIV viral load for $\geq 6$ months on a non-NRTI (NNRTI) or PI-based regimen [27,28]. Patients either continued the NNRTI (76\% received Atripla) or PI (80\% received either atazanavir or darunavir) or switched to Stribild. The 96-week results from both studies showed that coformulated Stribild was safe, effective at maintaining virologic suppression and reduced side effects attributed to the NNRTI or PI.

Genvoya has been evaluated in a switch study with a different study design in a unique patient population. Treatment-experienced patients with a history of 2-3 class drug resistance with at least two prior regimen failures and virally suppressed for $\geq 4$ months on a boosted darunavir-based regimen were randomized to continue current regimen or switch to Genvoya plus Darunavir $800 \mathrm{mg}$ daily [29]. Eligible patients were INSTI naive or had no history of INSTI resistance. At baseline, patients were taking a median of five tablets/day, $40 \%$ were taking more than 6 tablets/day and 65\% were taking at least one antiretroviral twice daily. Results at week 48 for this simplified, two tablet, once daily regimen in virally suppressed treatment-experienced patients demonstrated noninferiority to baseline regimens, safe, well tolerated, associated with greater patient satisfaction and improved adherence.

Another Genvoya switch study that is clinically relevant was studied in patients who had a documented M184V/I mutation on historical HIV genotype. The M184V/I is a common mutation that results in high-level resistance to lamivudine and emtricitabine, but increases susceptibility to tenofovir [30]. Therefore, tenofovir alafenamide may have additional activity against viruses with the M184V/I mutation. Previous switch studies with elvitegravir, coformulated as Stribild, were in patients with no previous virologic failure or resistance to emtricitabine. Study 1824 addressed the question of whether stable-suppressed patients with historic genotype showing M184V/I mutation can be switched to Genvoya. This was an open-label, single-arm study in 37 HIV-1-infected adults with HIV viral load less than 50 copies $/ \mathrm{ml}$ for at least 6 months and no prior virologic failure on PI or INSTI regimen who were switched to Genvoya. Results at week 24 showed all patients maintained HIV viral load less than 50 copies/ml and was well tolerated, thus supporting a switch to Genvoya in patients virologically suppressed on ART with preexisting M184V/I mutation [31]. However, these results are preliminary, the study is planned for 48 weeks and the numbers of patients enrolled is small, but this is encouraging data for a common clinical scenario.

In summary, elvitegravir coformulated in the single tablet once daily regimen Genvoya shows potent and rapid virologic suppression, excellent tolerability and significantly less renal and bone toxicity compared with its predecessor Stribild. Genvoya also has an advantage over other single tablet regimens, it has fewer side effects than Atripla, provides treatment for patients coinfected with hepatitis B and does not require HLA-B*5701 test recommended prior to starting the other INSTI single tablet regimen dolutegravir/lamivudine/abacavir (Triumeq ${ }^{\circledR}$ ). However, Genvoya like raltegravir, has seen its clinical role decline because of a low generic barrier to resistance and significant drug interactions due to cobicistat with coadministered medications. These two reasons are why Genvoya was downgraded on the DHHS treatment guidelines and no longer a preferred regimen for treatment naive patients. Genvoya is not recommended in pregnancy because of decreased elvitegravir and cobicistat plasma levels during third trimester, is not recommended for pediatrics weighing less than $25 \mathrm{~kg}$ or in patients with estimated creatinine clearance between 15 and $30 \mathrm{ml} / \mathrm{min}$ or estimated creatinine clearance less than $15 \mathrm{ml} / \mathrm{min}$ and not on hemodialysis [32]. Finally, the development and approval of the second-generation INSTI bictegravir (coformulated with emtricitabine and tenofovir alafenamide as Biktarvy ${ }^{\circledR}$ ) has essentially replaced the need once served by Genvoya. Both bictegravir and Genvoya are manufactured by the same pharmaceutical company, Gilead Sciences.

\section{Dolutegravir}

The INSTI that raised the bar and became the standard by which present, future and past antiretrovirals will be measured is dolutegravir (Tivicay ${ }^{\circledR}$ ). Dolutegravir was the third INSTI FDA approved (2013) and possesses all the beneficial characteristics of the INSTI class; durable virologic suppression, excellent tolerability, minimal adverse effects and efficacy in both treatment naive and treatment-experienced patients. What separates dolutegravir from the previous INSTIs discussed is the high genetic barrier to resistance and subsequent lack of virologic failure due to drug-resistant mutations. 
The clinical efficacy of dolutegravir in treatment naive patients was established in three clinical trials. The SINGLE trial was a pivotal study that compared dolutegravir + abacavir/lamivudine to Atripla [33]. The results from this randomized, double-blind, Phase III study in treatment naive patients showed that dolutegravir was superior $(\mathrm{p}=0.003)$ to Atripla. The difference in response between the two arms was driven by discontinuations due to adverse events associated with Atripla and not virologic failures. The difference in tolerability is not surprising due to the numerous and well documented CNS adverse effects associated with efavirenz [34]. Time to viral suppression (viral load $<50$ copies) was more rapid ( 28 vs 84 days) for dolutegravir $(\mathrm{p}<0.001)$ and is consistent with data from other INSTIs. What may be the most impressive data from this study is the lack of virologic failure due to the emergence of drug-resistant mutations. Data at 96 weeks reported no major treatment-emergent INSTI or NRTI resistance in patients receiving dolutegravir + two NRTIs [35]. The SPRING-2 study was a large safety and efficacy study that compared once daily dolutegravir versus twice daily raltegravir in treatment naive adults [36]. This randomized, double-blind, noninferiority study is the first head to head comparison of efficacy and safety of two INSTIs for treatment naive adults. Results from 96 weeks showed that dolutegravir was noninferior to raltegravir, both arms were well tolerated with few discontinuations, fewer patients had virologic failure with dolutegravir (5\%) compared with raltegravir $(10 \%)$ and there was no change in fasting lipids. Serum creatinine was mildly elevated (mean decrease in estimated creatinine clearance at week 48 was $16.5 \mathrm{ml} / \mathrm{min}$ ) due to dolutegravir's ability to inhibit renal organic cation transporter-1, which is the mechanism for mild increase in serum creatinine observed in dolutegravir clinical trials [37]. Similar to the SINGLE study, there was no evidence of treatment emergent resistance in patients with virologic failure on dolutegravir at week 96. The third pivotal study in treatment naive patients compared dolutegravir with the boosted PI darunavir/ritonavir. The FLAMINGO study is a multicenter, open-label, Phase IIIb, parallel group, noninferiority study that randomized treatment naive patients to receive dolutegravir or darunavir/ritonavir once daily + two NRTIs (either tenofovir/emtricitabine or abacavir/lamivudine) [38]. Results at 96 weeks showed that dolutegravir is well tolerated with few treatment discontinuations and had less effect on lipids compared with darunavir/ritonavir. Like the SINGLE study dolutegravir was found to be superior to darunavir/ritonavir. Differences in efficacy were primarily driven by lower rates of treatment withdrawal and higher rate of virologic response in patients with high baseline viral load (>100,000 copies $/ \mathrm{ml})$. Like the previous studies, no detection of antiviral drug resistance was seen in those patients with virologic failure. Taken together these studies laid the foundation for dolutegravir; a well-tolerated, very potent, with a high barrier to resistance, and in some studies superior to other antiretroviral regimens for treatment naive HIV-1-infected adults.

One advantage of dolutegravir is that it is available both as an individual tablet and a fixed dose single tablet regimen dolutegravir/lamivudine/abacavir (Triumeq). The benefit of a separate tablet is that it can be given with other drugs to be used in treatment-experienced patients with drug resistance. The SAILING study is a Phase III, randomized, double-blind, active-controlled, noninferiority study in antiretroviral experienced, INSTI-naive patients [39]. Those enrolled had resistance to two or more classes of antiretroviral drugs, but had to have 1-2 fully active drugs for background therapy. The results from this study demonstrated that once daily dolutegravir in combination with at least one other active drug provides superior virologic control $(\mathrm{p}=0.03)$ compared with raltegravir $400 \mathrm{mg}$ twice daily in treatment-experienced, INSTI-naive HIV-1-infected adults. Discontinuations were infrequent and adverse effects were similar for both groups. Those patients receiving dolutegravir had significantly less virologic failure compared with raltegravir. This study helped establish dolutegravir as the preferred INSTI in those patients who are INSTI naive, but have virologic failure on previous treatment regimens.

Another population where clinical data were lacking was in those patients who had failed an INSTI regimen with INSTI resistance. The VIKING-3 trial was designed to determine if dolutegravir has antiviral activity against raltegravir- and elvitegravir-resistant virus [40]. This single-arm study was open-label, Phase III that enrolled treatment experienced adult patients with documented INSTI resistance. Patients received dolutegravir $50 \mathrm{mg}$ twice daily plus optimized background therapy. Results at week 24 showed that $69 \%$ of subjects had achieved HIV viral load less than 50 copies/ml. Virologic nonresponders were most likely to have integrase mutations Q148+ 2 two additional INSTI mutations. Dolutegravir $50 \mathrm{mg}$ twice daily (double dose) was well tolerated and the results from this study resulted in FDA approval for patients with known or suspected HIV-1 INSTI resistance.

The clinical benefits have been well documented and impressive, however, recent reports of neural tube defects, unintentional weight gain and neuropsychiatric adverse events have raised concern with dolutegravir. Safety and pharmacokinetic data are instrumental in establishing guidelines for ART use in pregnancy. Collecting data on new drugs during pregnancy is challenging, but the story of dolutegravir illustrates why it is important and necessary. The DolPHIN-1 study randomized 60 women between 28-36 weeks gestation in South Africa or Uganda who 
received no antiretrovirals for the previous 6 months and were randomized to receive dolutegravir or efavirenz + two NRTIs [41]. The thought was women who present late in pregnancy would achieve undetectable viral loads more rapidly with dolutegravir compared with efavirenz. This study also looked at dolutegravir pharmacokinetics in the third trimester. This is relevant because other drugs are known to have lower plasma levels in the third trimester [42]. Results from this study and one other showed that dose adjustment for dolutegravir would not be necessary and a more rapid decline in HIV-1 viral load was observed for dolutegravir, which might have clinical benefits during pregnancy [43].

In comparison, the Tsepamo cohort initially observed a signal of an increased risk of neural tube defects when dolutegravir was used at the time of conception compared with nondolutegravir antiretroviral regimens [44]. The key findings were that all the birth defects $(n=4)$ were observed in women exposed to dolutegravir at the time of conception. The study reported a rate of neural tube defects of approximately $0.9 \%$ compared with $0.1 \%$ risk among women taking nondolutegravir-based regimens at the time of conception. No evidence was reported of neural tube defects in women who started dolutegravir during pregnancy. These interim data were enough to prompt the DHHS treatment guidelines to recommend that dolutegravir should not be prescribed for women who are pregnant or within 12 weeks of postconception, women who are of child-bearing potential and planning on becoming pregnant, and women of childbearing potential who are sexually active and not using effective contraception $[1,45]$.

The safety of dolutegravir during pregnancy has been one of the most urgent questions in global HIV care in the past year. As a result, some countries have placed dolutegravir-based regimens on hold as preferred therapy. Follow-up data were recently published and presented at the 10th IAS Conference on HIV Science 2019. The recent data presented from the Tsepamo cohort showed that the prevalence of neural tube defects among women who received dolutegravir at conception was lower than initially reported, but still slightly higher than other exposure groups $(0.3 \%$ dolutegravir vs $0.1 \%$ nondolutegravir ART) [46,47]. Based on these current findings and other dolutegravir data in pregnancy, the WHO updated their guidelines to recommend the use of dolutegravir as the preferred first-line and second-line treatment for all populations, including pregnant women and those of childbearing age [48]. This recommendation is based on the most recent data where the risk of neural tube defects are significantly lower than initial data suggested and the benefits of dolutegravir outweigh the risk. However, the guidelines acknowledge that more data are needed to confirm or refute this early signal and emphasize the need for continued monitoring of the risks of neural tube defects and support the education for women to make an informed choice regarding treatment with dolutegravir.

In the early ART era, weight gain was not uncommon and thought to be a sign of nutritional recovery resulting from virologic suppression and immune reconstitution. Some recent reports have observed significant weight gain (3-6 kg at 9-18 months) in patients virally suppressed who were switched to INSTIs or initiating an INSTI-based regimen, however, the weight gain was particularly evident in those patients on dolutegravir [4951]. Weight gain is not a metric that is typically assessed in HIV clinical trials. The etiology of weight gained observed in patients on dolutegravir based regimens needs further study. Whether this observation is an INSTIclass effect or more pronounced with dolutegravir remains to be elucidated. Another postmarketing adverse events reported are neuropsychiatric events attributed to dolutegravir. A few observational cohorts have reported on dolutegravir discontinuations that appear to be higher than those observed in randomized clinical trials [52-54]. The discontinuations are most often described as neuropsychiatric that include insomnia/sleep disturbances, anxiety and depression and appear to be associated with dolutegravir and not raltegravir or elvitegravir. The overall rates of discontinuations are low and variable depending on the cohort. The data support the continued pharmacovigilance of postmarketing observations and support further investigations of neuropsychiatric adverse effects associated with dolutegravir and INSTIs as a class.

Despite the recent observations of weight gain, neuropsychiatric adverse effects and the initial signal of neural tube defects in pregnant women exposed to dolutegravir at the time of conception, dolutegravir remains a valuable agent in the HIV treatment arsenal. Dolutegravir has shown superior efficacy compared with other antiretroviral agents, excellent tolerability with few discontinuations in clinical trials, minimal drug interactions and a high barrier to drug resistance. Dolutegravir can be prescribed for both treatment naive and experienced patients and in some patients with INSTI-associated mutations. As dolutegravir gains traction in developing nations with a high prevalence of mycobacterium tuberculosis, no dose adjustment is necessary when coadministered with rifabutin and a dose increase of $50 \mathrm{mg}$ twice daily is recommended if coadministered with rifampin [55]. Dolutegravir can be used in children age 12 years or older and weigh more than $40 \mathrm{~kg}$ and does not require dose adjustment 
in mild-moderate hepatic failure or renal impairment including dialysis [55]. Unlike previous INSTIs discussed, dolutegravir continues to remain prominent in clinical practice today.

\section{Bictegravir}

In 2018, the FDA approved a fourth INSTI, bictegravir, which is only available as a fixed-dose single tablet regimen of bictegravir/emtricitabine/tenofovir alafenamide (Biktarvy). Bictegravir is similar in structure to dolutegravir and shares many of the same characteristics of dolutegravir such as excellent potency, long half-life allowing for once daily dosing, well tolerated, minimal drug interactions and most importantly a high genetic barrier to drug resistance. The advantage of Biktarvy over Genvoya is that it does not require a pharmacokinetic booster, therefore significantly decreasing potential drug interactions. The other advantage is bictegravir has a higher genetic barrier to resistance compared with elvitegravir. Biktarvy is indicated for treatment naive or to replace current regimen in patients virologically suppressed (HIV viral load $<50$ copies $/ \mathrm{ml}$ ) on a stable ART regimen for at least 3 months with no history of treatment failure and no known drug mutations to any of the components of Biktarvy. Unlike dolutegravir, Biktarvy is not indicated for treatment-experienced patients or those who have failed previous INSTIbased regimens. However, that has not deterred clinicians from using Biktarvy in patients with previous NRTI resistance based on in vitro and clinical data [56,57]. Similar to Study 1824 (Genvoya study), pre-existing drug resistance did not affect Biktarvy efficacy through week 48 in a pooled analysis of baseline resistance from Phase III clinical trials in virologically suppressed patients switching to Biktarvy [58,59]. Pre-existing genotypes were obtained using proviral archive genotypes and no significant difference in virologic outcomes were observed by the presence or absence of archived resistance in the Biktarvy group, including 52/54 patients who remained virologically suppressed despite archived M184V/I mutation [57]. These results provide more data that Biktarvy is an effective treatment option for virologically suppressed patients with evidence of archived M184V/I mutation that is not detected on routine HIV genotype.

Biktarvy was evaluated in two large randomized, double-blind, active-controlled, multicentered noninferiority trials completed in treatment-naive patients. One study compared Biktarvy with Triumeq, 96-week results showed Biktarvy was noninferior to Triumeq with 88 versus $90 \%$ achieving HIV viral load less than 50 copies/ml [60]. The only significant difference was more nausea was reported in the Triumeq arm (17\% attributed to study drug Triumeq compared with $6 \%$ for Biktarvy, $\mathrm{p}<0.0001)$. The other study compared Biktarvy with dolutegravir + emtricitabine/tenofovir alafenamide (each with matching placebo) and results also showed noninferiority [61]. Both studies showed no difference in treatment outcomes, regimens were well-tolerated with few discontinuations, and no patient developed treatment emergent resistance to any study drug.

Biktarvy was also evaluated in two large randomized, controlled, Phase III switch studies. These were similarly designed trials where patients were virally suppressed on boosted PI or dolutegravir-based regimen and randomized to continue current regimen or switch to Biktarvy [58,59]. Results from both studies showed virologic suppression was maintained when switching to Biktarvy. Besides maintaining efficacy both studies showed the overall incidence and severity of adverse events were similar between groups, there were few discontinuations and no patient developed treatment emergent resistance to any component of Biktarvy. Changes from baseline total cholesterol, LDL cholesterol and triglycerides were similar between groups except for patients receiving abacavir/lamivudine + boosted PI. In this arm, those patients who switched to Biktarvy showed a significant decrease in total cholesterol, LDL and triglycerides.

Bictegravir, like its predecessor dolutegravir, is potent, well tolerated, has minimal drug interactions, high genetic barrier to drug resistance and DHHS recommended preferred treatment for HIV-1 treatment naive adults. For these reasons, Biktarvy is widely prescribed in clinical practice at this time. Biktarvy has the additional benefit of activity against hepatitis B and is smaller than other coformulated single tablet INSTI regimens. However, longterm clinical data are not established, whether or not bictegravir has similar clinical adverse effects as dolutegravir or other adverse effects not yet recognized remains to be determined. Biktarvy is not recommended in patients with severe hepatic impairment, creatinine clearance less than $30 \mathrm{ml} / \mathrm{min}$, pregnancy or in pediatric patients less than 18 years of age [62].

\section{Cabotegravir}

Zidovudine was the first antiretroviral FDA approved for HIV-1 treatment in 1987, there are now over 30 medications approved for HIV-1 treatment. The impact of ART on survival and quality of life changed the course of the epidemic from a fatal disease to one that is managed like other chronic diseases. Life expectancy for 
people living with HIV is fast approaching that of the HIV-negative population [63]. What almost all antiretroviral therapies have in common are oral formulations that must be taken daily and require a high level of adherence to maintain virologic suppression and to prevent the development of drug resistance. However, the treatment paradigm is changing. Cabotegravir is an INSTI in late clinical development that has a long half-life and the chemical properties that allow it to be formulated into a long-acting nanosuspension for intramuscular injection (IM). Cabotegravir is being studied in combination with a long-acting preparation of rilpivirine (NNRTI) as a two-drug regimen for adults on stable ART who want to switch therapy. Long-acting cabotegravir is also ideal for HIV prevention and is currently being studied for PrEP. Cabotegravir will be a welcomed addition for those patients who do not want to take pills daily. Both HIV treatment and PrEP require high rates of daily medication adherence to be successful, the addition of long-acting cabotegravir will change the accepted treatment philosophy of lifelong daily pills.

Cabotegravir is similar in structure to dolutegravir and shares some of the same beneficial attributes such as high barrier to resistance, excellent potency, minimal drug interactions, no impact on CYP450 enzymes and does not require dose adjustment in patients with renal impairment not on dialysis. What distinguishes cabotegravir from dolutegravir are physiochemical properties and a pharmacokinetic profile characterized by a long half-life and it can be formulated as a nanoparticle for injection. Cabotegravir also does not affect renal tubular secretion of creatinine like dolutegravir. Oral cabotegravir has an elimination half-life of approximately $40 \mathrm{~h}$ compared with $13 \mathrm{~h}$ for dolutegravir. The formulation of cabotegravir as a long-acting depot nanosuspension has an elimination half-life of 25-54 days [64]. Plasma levels exceed protein adjusted $\mathrm{IC}_{90}$ for approximately 16 weeks following a single dose of $800 \mathrm{mg}$ IM injection [65]. Phase I clinical studies showed prolonged exposures of at least 30 days following gluteal IM injections for both cabotegravir and rilpivirine, thus allowing for monthly or longer dosing intervals [64,65].

The journey for cabotegravir started with the LATTE trial that was designed as an induction/maintenance study to establish efficacy and tolerability of the two-drug oral combination of cabotegravir + rilpivirine [66]. The induction phase of the study compared three different doses of oral cabotegravir + two NRTIs to efavirenz + two NRTIs (standard of care) for 24 weeks. Those patients in the cabotegravir arms virologically suppressed at week 24 were switched to an NRTI sparing two-drug regimen of oral cabotegravir + oral rilpivirine. The results showed excellent virologic suppression at both 24 weeks (induction) and 48 weeks (maintenance) and provided proof of principle of two-drug maintenance treatment. Oral cabotegravir 30-mg dose was selected to move forward based on the results of this study.

The positive results from LATTE led to the LATTE-2, also designed as induction/maintenance study for treatment naive patients who were initiated on oral cabotegravir + abacavir/lamivudine (conventional triple therapy), at week 16 oral rilpivirine was added for 4 weeks [67]. The oral lead-in dosing strategy is necessary for both long-acting drugs to ensure that patients can tolerate both drugs before receiving the long-acting dose. At week 20, patients were randomized to continue oral therapy or switch to two different intramuscular injection doses of long-acting cabotegravir + long-acting rilpivirine. Results at 96 weeks showed similar virologic efficacy between the oral dose and both IM injection arms of the study, however, the 400-mg dose of cabotegravir +600 -mg rilpivirine administered every 4 weeks was chosen for Phase 3 studies. Although both 4 - and 8-week groups maintained virologic suppression, there were a few more patients in the 8-week group that were virologic nonresponders (HIV-1 RNA > 50 copies/ml) as defined by the FDA snapshot algorithm, which led to the selection of the 4-week regimen for future Phase III clinical studies [67]. Pharmacokinetic analysis at week 48 showed geometric mean trough concentrations for the 4-week group and the 8-week group were 16-times and nine-times greater than the in vitro protein-adjusted $90 \%$ inhibitory concentration against wild-type HIV-1. As for rilpivirine, week 48 trough values were eight- and five-times greater than protein adjusted $\mathrm{IC}_{90}$ for the 4- and 8-week groups, respectively. Regarding safety and patient satisfaction, there were few discontinuations, $99 \%$ of injection site reactions were grade 1 (84\%) or grade $2(15 \%)$ and these reactions decreased over time with only $29 \%$ complaining of injection site reactions by week 48. Patients reported high levels of treatment satisfaction with the injectable regimens, preferred them to the oral regimen and wanted to continue with IM treatment. These favorable results led to the Phase III evaluation of long-acting cabotegravir + long-acting rilpivirine administered as an IM injection.

ATLAS study is a multicenter, randomized, open-label, Phase III, noninferiority switch study that compared patients with no prior virologic failure on a stable regimen with HIV viral load less than 50 copies $/ \mathrm{ml}$ to continue ART ( $\mathrm{n}=308)$ or switch to oral cabotegravir $30 \mathrm{mg}+$ oral rilpivirine $25 \mathrm{mg}(\mathrm{n}=308)$ for 4 weeks and then receive monthly IM (ventrogluteal) injections of cabotegravir $400 \mathrm{mg}+$ rilpivirine $600 \mathrm{mg}$ [68]. Results at 48 weeks showed noninferiority with more than $90 \%$ of patients in both arms virally suppressed. Long-acting cabotegravir + long- 
acting rilpivirine was well tolerated with few treatment discontinuations. Although injection site reactions were common, they were mild, $90 \%$ of participants scored injection site reactions and $86 \%$ scored pain as totally or very acceptable [69]. Patients also preferred the switch to injections rather than continue baseline oral regimens.

The FLAIR study was slightly different than ATLAS, but the results were similar. The induction phase for FLAIR enrolled treatment naive patients who initiated Triumeq for 20 weeks and then switched to maintenance phase of oral cabotegravir $30 \mathrm{mg}$ daily + rilpivirine $25 \mathrm{mg}$ daily for 4 weeks and then long-acting cabotegravir $400 \mathrm{mg}$ IM + long-acting rilpivirine $600 \mathrm{mg}$ IM every 4 weeks or continue with Triumeq [70]. Results at 48 weeks showed similar efficacy with both arms ( $>90 \%$ had HIV viral load $<50$ copies $/ \mathrm{ml}$ ). Similar to the ATLAS study, oral and IM injections were well tolerated with few discontinuations and injection site reactions were mild. Also consistent with the ATLAS study was patient reported satisfaction was higher in those receiving the injection and they preferred injections over their prior oral therapy. If the FDA approved this two-drug injectable regimen, it would give patients 1 month between doses, thus changing HIV treatment from 365 days per year to just 12 .

The efficacy of daily oral emtricitabine/tenofovir disoproxil (Truvada) for PrEP has been evaluated in clinical trials [71,72]. In 2012, the FDA approved Truvada for PrEP, however, poor adherence and potential for drug toxicity are two factors that have limited the implementation of preventing new HIV-1 infections in persons at risk. Longacting cabotegravir is a potential alternative treatment for PrEP because barriers such as high level of adherence to daily Truvada, potential for renal and bone toxicity and the inconvenience of daily dosing make cabotegravir an attractive alternative. Population pharmacokinetic modeling suggest that 800-mg dose administered every 12 weeks should establish plasma concentrations expected to be protective against HIV-1 infection (four-times the protein adjusted $\left.\mathrm{IC}_{90}\right)[64,65]$.

Long-acting cabotegravir has been studied in two placebo-controlled, randomized, double-blind, Phase IIa safety and tolerability clinical PrEP trials, ÉCLAIR and HPTN 077. The ÉCLAIR trial was a multicentered, double-blind, randomized (5:1), placebo-controlled trial that enrolled $127 \mathrm{HIV-negative} \mathrm{men} \mathrm{who} \mathrm{have} \mathrm{sex} \mathrm{with}$ men [73]. Participants received oral cabotegravir $30 \mathrm{mg}$ lead-in dose or placebo for 4 weeks. This was followed by 1 week washout period and after safety assessment received three long-acting cabotegravir 800-mg IM injections (ventrogluteal) every 12 weeks or saline placebo. The results from this study showed that long-acting cabotegravir was well tolerated (only four participants discontinued study drug) with mild-to-moderate pain from injection site reactions. Patients reported that injection pain was tolerable, prefer to continue with injections, and would recommend the treatment to others. Participants also did not encounter problems adhering to 12 -week injection schedule and prefer the injection over daily oral dosing. However, pharmacokinetic analysis showed lower than expected cabotegravir levels and therefore a different dosing strategy of $600 \mathrm{mg}$ administered every 8 weeks will be pursued. Mean trough concentrations were approximately half of expected values. Lower levels were thought to be due to faster than anticipated absorption from the depot site resulting in higher peaks and lower trough concentrations. Some individuals had inconsistent concentration-time profiles across the 12 -week dosing interval suggesting variable absorption rates between injections. This might be due to injection technique or other unknown factors. Although no individual seroconverted while on study drug prolonged low-level cabotegravir concentrations suggest a theoretical risk for the development of drug resistance should HIV infection occur. Also of concern is will HIV seroconversion occur when cabotegravir levels are below some critical not yet determined threshold in persons who miss doses or stop treatment? Because the $800-\mathrm{mg}$ dose administered every 12 weeks resulted in suboptimal exposure, $600 \mathrm{mg}$ administered every 8 weeks will be studied for safety, acceptability and pharmacokinetics.

HPTN 077 was a double-blind, placebo-controlled Phase IIa trial similar to ECLAIR [74]. However, when the pharmacokinetic analysis from ÉCLAIR was completed, a second cohort was added to HPTN 077. Cohort 1 was similar to ÉCLAIR where long-acting cabotegravir $800 \mathrm{mg}$ or saline placebo was administered every 12 weeks (for a total of three injections). Cohort 2 administered long-acting cabotegravir $600 \mathrm{mg}$ or saline placebo every 8 weeks (the first two injections were separated by 4 weeks). The study enrolled both HIV-negative males and females who were randomized to receive 4 week lead-in oral dosing or placebo followed by either saline placebo or long-acting cabotegravir injections. Results from Cohort 1 confirmed the pharmacokinetic results from ÉCLAIR. Males in particular did not consistently achieve the protein adjusted $\mathrm{IC}_{90}$ goals compared with females. Results also showed that no safety concerns were found, injection site reactions were common, but rarely led to discontinuations and that pharmacokinetic analysis support cabotegravir 600-mg IM every 8 weeks for PrEP. The positive results from this study have led to Phase III studies that are currently enrolling and will be evaluating the $600 \mathrm{mg}$ dose every 8 weeks. 
Long-acting cabotegravir has brought a new intervention for both HIV-1 treatment and prevention. Injectable cabotegravir and rilpivirine offer a trade-off from the inconvenience of oral medications to the inconvenience of uncomfortable monthly injections. The logistical concerns for administering ventrogluteal IM injections will need to be resolved in the clinic. Patient transportation for injection administration, establishing regular monthly injection appointments and clinic access to drug will all need to be addressed to optimize the utility of this drug. Globally, storage of long-acting cabotegravir and rilpivirine requires refrigeration, which will limit its availability and data in pregnancy are yet to be determined. Also, baseline resistance testing will be important prior to initiating therapy because of the potential for transmitted resistance to rilpivirine. The upside is that a recent press release reported on positive results from the ATLAS-2M trial [75]. This study compared long-acting cabotegravir + longacting rilpivirine administered every 4 versus 8 weeks in virally suppressed patients. Results at 48 weeks showed noninferiority with the added benefit of potentially further reducing the number of injections to 6 per year. Follow-up data from this important trial are eagerly awaited.

\section{Changing the HIV treatment paradigm}

Standard of care since the introduction of highly active ART for HIV-1 treatment has always been two NRTIs + a third agent. However, as HIV patients are living longer, there is a concern regarding cumulative drug exposure and long-term toxicities. Would the patient benefit over a lifetime if subjected to fewer drugs? Two-drug therapy for HIV is not new, some studies were done over a decade ago, but the results have been mixed $[76,77]$. The advantage of two-drug regimens would decrease lifetime drug exposure and potential long-term toxicities, as well as, reduce cost. The concerns surrounding two-drug regimens will be durability, efficacy and treatment-emergent resistance.

The properties of dolutegravir and rilpivirine make them good candidates for two-drug therapy. They both have long half-lives, neither require pharmacokinetic boosting, they have minimal drug interactions, excellent tolerability and low dose for coformulation. The SWORD-1 and SWORD-2 were open-label, parallel group, multicenter, Phase III, randomized noninferiority switch studies evaluating efficacy and safety of once daily dolutegravir $50 \mathrm{mg}+$ rilpivirine $25 \mathrm{mg}$ versus current antiretroviral regimen [78]. Enrolled participants were virally suppressed for greater than 6 months, had no history of virologic failure and not infected with hepatitis B virus. The pooled analysis at 48 weeks showed that the two-drug regimen was noninferior to the traditional three-drug regimen. Results also indicated that dolutegravir + rilpivirine maintained virologic suppression (95\%) with no increased risk of developing drug resistance. There were more reported adverse effects in the two-drug arm, which is expected because patients switched from a stable regimen to two new drugs. However, no unexpected adverse effects were identified with the two-drug combination and there was no effect on lipids. In November 2017, the FDA approved the coformulation of $50 \mathrm{mg}$ dolutegravir $/ 25 \mathrm{mg}$ rilpivirine (Juluca ${ }^{\circledR}$ ) as the first two-drug-coformulated tablet that provides virologically suppressed patients the option to switch from a three drug regimen. The potential benefit is avoiding NRTI cumulative toxicities, lipid neutral and has minimal drug interactions. One limitation is that Juluca is recommended to take with food to maximize absorption and acid-reducing agents should be avoided.

The next development for two-drug therapy is head-to-head comparison with DHHS guideline recommended three-drug regimen in treatment naive patients. For this, dolutegravir would be partnered with lamivudine (FDA approved in 1995), an NRTI with a long history of excellent safety, efficacy and tolerability. The GEMINI-1 and GEMINI-2 were two large identically designed, multicentered, double-blind, randomized, noninferiority, Phase III trials in HIV-1 treatment naive patients [79]. Patients were randomized to receive dolutegravir + lamivudine or dolutegravir + Truvada (DHHS recommended three-drug regimen). Results at 48 weeks showed noninferior virologic efficacy and most notable neither arm was associated with emergence of drug resistance. Results also showed a rapid decline in HIV viral load in both arms, comparable virologic efficacy observed in patients with high viral load (>100,000 copies $/ \mathrm{ml})$, and safety and tolerability were similar between groups. At the 10th IAS Conference on HIV Science 2019, 96-week data were presented on GEMINI-1 and GEMINI-2. The two-drug regimen of dolutegravir + lamivudine continued to have virologic efficacy noninferior to three-drug regimen dolutegravir + Truvada (HIV viral load $<50$ copies $/ \mathrm{ml}, 86$ vs $89.5 \%$, respectively) [80]. Less than $1.5 \%$ of patients developed virologic failure in either arm and still no treatment-emergent integrase or NRTI mutations were detected. Safety and tolerability also continue to remain similar between groups. In April 2019, the FDA approved the two-drug combination tablet dolutegravir/lamivudine (Dovato ${ }^{\circledR}$ ), the first two-drug regimen for treatment naive HIV-1-infected adults without hepatitis B coinfection. Whether or not a treatment shift to two-drug therapy will take root in in clinical practice remains to be determined. 


\section{Resistance}

INSTIs have demonstrated superior efficacy and excellent tolerability in clinical trials, however, the emergence of drug-resistant virus can result in virologic failure. Consistent with other antiretroviral drug classes, HIV has shown the ability to develop primary-resistant mutations within the HIV integrase catalytic site. Although drug-resistant mutations for second-generation INSTIs (i.e., dolutegravir and bictegravir) are rare.

First-generation INSTIs (i.e., raltegravir and elvitegravir) are characterized by a low genetic barrier to resistance and extensive cross-resistance. Results from clinical trials and site-directed mutagenesis experiments identify three major primary resistance pathways for raltegravir are N155H, Q148R/H/K and Y143R/C [18,81]. Raltegravir is also associated with the development of secondary mutations that continue to evolve under continuous drug pressure. In some instances, initial (primary) mutations are replaced by different mutational combinations that are associated with increased resistance. Secondary mutations associated with raltegravir resistance are G140S/A, E138A/K, L74M, E92Q, T97A, U151I and G163R [82]. The combination Q148, which sits in the critical part of the enzyme active site, plus G140, which improves enzyme function that is lost due to Q148 mutation, represents the highest level of resistance to raltegravir and significant cross resistance to all other INSTIs, but restores viral fitness [83].

Elvitegravir primary resistance has been shown at six integrase positions: T66I/A/K, E92Q/G, T97A, S147G, Q148R/H/K and N155H [84]. Both raltegravir and elvitegravir share similar binding properties within HIV integrase catalytic site and thus not surprising both exhibit overlapping primary resistance profiles. Compare that to second-generation INSTIs where structural analysis of dolutegravir shows that it is able to make better contact with viral DNA and is able to readjust its position and conformation to structural changes in the catalytic site of raltegravir- and elvitegravir-resistant virus. The effect is slower dissociation and may explain why highlevel resistance to dolutegravir and bictegravir is uncommon and rarely documented in clinical trials [85]. However, reduced susceptibility to dolutegravir has been observed in patients with Q148 plus two or more secondary mutations and low level resistance (twofold, fivefold) was reported with R263K and G118R mutations, respectively [40,86]. Due to the perceived high genetic barrier to resistance and potency dolutegravir monotherapy was compared with Triumeq ${ }^{\circledR}$ (traditional three-drug therapy) in virologically suppressed patients. The intent was that simplified regimens might reduce long-term drug toxicity and cost less. Results at 24 weeks were impressive, however, results at 48 weeks showed that the incidence of virologic failure was $9.7 \%$ in the dolutegravir monotherapy arm compared with $0 \%$ in the Triumeq arm [87]. The increased risk of virologic failure over time and the identification of a few patients developing INSTI resistance suggest that dolutegravir monotherapy should be avoided.

Similar to dolutegravir, bictegravir retains activity against primary raltegravir and elvitegravir mutations. Bictegravir also has reduced susceptibility, but to a lesser degree compared with dolutegravir in the presence of Q148 plus one or two additional mutations [88]. In vitro data from a panel INSTI-resistant mutants showed that most of the isolates were more susceptible to bictegravir compared with dolutegravir or cabotegravir [89]. Cabotegravir-resistance profile is still developing, the comparative genetic barrier to resistance for patient-derived integrase-resistant isolates was raltegravir $>$ elvitegravir $>$ cabotegravir $>$ dolutegravir and bictegravir [90]. Cabotegravir has demonstrated excellent activity against a broad range of viruses from various clades across geographic locations; however, results from Phase III clinical trial (FLAIR) identified three patients with virologic failure with clade A-1 virus. Whether or not this specific clade is less susceptible to cabotegravir needs further clarification [91].

Unlike other classes of antiretrovirals, transmitted INSTI resistance is extremely rare. Cohort studies in the USA and Europe have reported on the prevalence of INSTI transmitted drug resistance to be $0-0.1 \%$ [92]. There have been only a few cases reported in the literature and because the reported prevalence is so low baseline HIV integrase testing is not routinely recommended for HIV patients entering care $[1,93]$ As INSTI use continues to increase globally, the prevalence of transmitted INSTI resistance may increase, which would call for a re-evaluation of the current guidelines for HIV+ patients entering care.

\section{Conclusion}

The development of INSTIs have had a lasting and significant impact on ART. Nowhere is this more evident than in DHHS treatment guidelines where INSTIs anchor the preferred regimens recommended for HIV-1-infected adults. The crown jewels of the class are dolutegravir and bictegravir, what makes them so favorable is their antiviral potency, minimal drug interactions, excellent tolerability, once daily coformulated single tablet regimens, approved for treatment naive and treatment-experienced patients (dolutegravir), and most importantly their high barrier to drug resistance. Vigilant monitoring needs to continue for dolutegravir regarding the early signal of neural tube 
defects, unintentional weight gain and neuropsychiatric side effects to determine if this is an INSTI class effect or unique to dolutegravir. We look forward to these data to provide better education for patients and medical providers. INSTIs have changed the treatment paradigm as we have known it for the past 20 years with two-drug therapy challenging traditional three-drug therapy. On the horizon is long-acting cabotegravir, which is also primed to change the treatment paradigm by offering 4- and 8-week injections instead of daily pills for both treatment and PrEP. By the end of this decade, INSTIs will have a significant role for every indication for HIV treatment and prevention. From a clinical standpoint, it will be challenging to improve upon this class of antiretrovirals, what remains elusive is a cure.

\section{Future perspective}

Over the next 5-10 years, Bictarvy, due to its excellent characteristics, will likely be the single tablet regimen that the majority of treatment naive patients will be prescribed. For those patients not willing or tired of taking a daily pill long-acting cabotegravir plus long-acting rilpivirine will be and excellent alternative. Especially if the formulation will allow for longer than monthly injections, consider a goal of receiving only six doses or fewer per year. Improving acceptance and convenience in clinical practice could be achieved with the development of a long-acting formulation that allows for subcutaneous or deltoid injection instead of gluteal. Two-drug ART will become accepted and with long-term data will be elevated to a preferred treatment option on guidelines for treatment naive persons. Finally, the availability of more generic antiretroviral medications and combinations may challenge treatment guidelines when cost may play a larger role for healthcare systems and developing countries.

\section{Executive summary}

\section{Background}

- Over the last decade, integrase strand transfer inhibitors (INSTIs) have had a significant effect on HIV-1 treatment; however, despite excellent clinical efficacy and excellent tolerability, this class of drugs has clinical concerns that should not be ignored.

Mechanism of action

- This class of antiretrovirals interferes with the two-step process of insertion of viral DNA into host DNA. This is accomplished by inhibition of HIV-1 integrase enzyme.

\section{Raltegravir}

- The first INSTI FDA approved was shown to be effective in both treatment naive and experienced patients. However, because of twice daily dosing and low genetic barrier to resistance the impact of this drug was not long lasting.

Elvitegravir

- First INSTI that was part of a single tablet regimen, however, because it required cobicistat to inhibit hepatic metabolism and had a low genetic barrier to resistance its clinical utility waned.

Dolutegravir

- The first of the second generation of INSTI that not only showed superiority over other antiretroviral agents but also has a high genetic barrier to resistance, thus becoming one of the gold standard INSTIs that all antiretrovirals will be compared with.

\section{Bictegravir}

- Second-generation INSTI very similar to dolutegravir and coformulated as a single tablet regimen that is preferred agent on treatment guidelines.

Cabotegravir

- Long-acting INSTI in late clinical development that will remove the burden of daily dosing and has data in treatment naive and pre-exposure prophylaxis patients.

Changing the HIV treatment paradigm

- Two-drug therapy anchored by dolutegravir is challenging the way we have traditionally treated HIV infection. Resistance

- Like all antiretorvirals, HIV has developed resistant mutations to INSTIs. Some drugs in this class are more susceptible to development of resistance than others.

Conclusion

- INSTIs have and will continue to have a lasting and significant impact on HIV-1 treatment. 


\section{Open access}

This work is licensed under the Attribution-NonCommercial-NoDerivatives 4.0 Unported License. To view a copy of this license, visit http://creativecommons.org/licenses/by-nc-nd/4.0/

Financial \& competing interests disclosure

The author has no relevant affiliations or financial involvement with any organization or entity with a financial interest in or financial conflict with the subject matter or materials discussed in the manuscript. This includes employment, consultancies, honoraria, stock ownership or options, expert testimony, grants or patents received or pending, or royalties.

No writing assistance was utilized in the production of this manuscript.

\section{References}

Papers of special note have been highlighted as: $\bullet$ of interest; $\bullet \bullet$ of considerable interest

1. Panel on Antiretroviral Guidelines for Adults and Adolescents. Guidelines for the use of antiretroviral agents in adults and adolescents with HIV. Department of Health and Human Services. What to start: initial combination regimens for antiretroviral-naïve patient (Table 6A). http://aidsinfo.nih.gov/contentfiles/lvguidelines/AdultandAdolescentGL.pdf

-. The Department of Health and Human Services adult treatment guidelines are the in-depth overview for primary care clinicians on the management of HIV infection.

2. Craigie R. HIV integrase, a brief overview from chemistry to therapeutics. J. Biol. Chem. 276, 23213-23216 (2001).

3. Engleman A, Mizuuchi K, Craigie R. HIV-1 DNA integration: mechanisms of viral DNA cleavage and DNA strand transfer. Cell 67, 1211-1221 (1991).

4. Ellison VH, Abrams TR, Lifson J, Brown P. Human immunodeficiency virus integration in a cell free system. J. Virol. 64, 2711-2715 (1990).

5. Engleman A, Craigie R. Identification of conserved amino acid residues critical for human immunodeficiency virus type 1 integrase function in vitro. J. Virol. 66, 6361-6369 (1992).

6. Markowitz M, Morales-Ramirez JO, Nguyen BY et al. Antiretroviral activity, pharmacokinetics, and tolerability of MK-0518, a novel inhibitor of HIV-1 integrase, dosed as monotherapy for 10 days in treatment-naïve HIV-1 infected individuals. J. Acquir. Immune Defic. Syndr. 43(5), 509-515 (2006).

7. Steigbigel RT, Cooper DA, Kumar PN et al. Raltegravir with optimized background therapy for resistant HIV-1 infection. N. Engl. J. Med. 359, 339-354 (2008).

8. Steigbigel RT, Cooper DA, Teppler $\mathrm{H}$ et al. Long-term efficacy and safety of raltegravir combined with optimized background therapy in treatment-experienced patients with resistant HIV infection: week 96 results of the BENCHMRK 1 and 2 Phase III trials. Clin. Infect. Dis. 50, 605-612 (2010).

9. Lennox JL, DeJesus E, Lazzarin A et al. Safety and efficacy of raltegravir-based versus efavirenz-based combination therapy in treatment-naïve patients with HIV-1 infection: a multicenter, double-blind randomized controlled trial. Lancet 374, 796-806 (2009).

10. Lake JE, Currier JS. Switching antiretroviral therapy to minimize metabolic complications. HIV Ther. 4, 693-711 (2010).

- Describing the benefits of switching antiretroviral therapy (ART) and establishes the principles for why the clinical trial design is so popular now.

11. Eron JJ, Young B, Cooper DA et al. Switch to a raltegravir-based regimen versus continuation of a lopinavir-ritonavir-based regimen in stable HIV-infected patients with suppressed viremia (SWITCHMRK 1 and 2): two multicenter, double-blind, randomized controlled trials. Lancet 375, 396-407 (2010).

- First switch study that failed, this study is important because it identified previous background drug resistance as the cause. This characteristic would be important for the design of future ART switch studies.

12. Merck. Isentress US Prescribing Information. Merck \& Co., Inc., NJ, USA. www.merck.com/product/usa/pi_circulars/i/isentress/isentress_pi.pdf

13. CDC. Updated Guidelines for Antiretroviral Postexposure Prophylaxis after Sexual, Injection Drug Use, or other non-occupational Exposure to HIV- United States, 2016. From the Centers of Disease Control and Prevention, U.S. Department of Health and Human Services. www.cdc.gov/hiv/pdf/programresources/cdc-hiv-npep-guidelines.pdf

14. New York State Department of Health AIDS Institute Clinical Guidelines Program. ART regimens for oPEP. www.hivguidelines.org/pep-for-hiv-prevention/occupational/\#tab_6

15. Shamsuddin H, Raudenbush CL, Sciba BL et al. Evaluation of neural tube defects (NTD) after exposure to raltegravir during pregnancy. J. Acquir. Immune Defic. Syndr. 81(3), 247-250 (2019).

16. Blonk MI, Colbers AP, Hidalgo-Tenorio C et al. Raltegravir in HIV-1 infected pregnant women: pharmacokinetics, safety, and efficacy. Clin. Infect. Dis. 61(5), 809-816 (2015). 
17. Croce F, Vitello P, Dalla Pria A et al. Severe raltegravir-associated rhabdomyolysis: a case report and review of the literature. Int. J. STD AIDS 21, 783-785 (2010).

18. Blanco JL, Varghese V, Rhee SY et al. HIV-1 integrase inhibitor resistance and its clinical implications. J. Infect. Dis. 203, 1204-1214 (2011).

19. Nathan B, Bayley J, Waters L, Post FA. Cobicistat: a novel pharmacoenhancer for co-formulation with HIV protease and integrase inhibitors. Infect. Dis. Ther. 2, 111-122 (2013).

20. Lepist EI, Zhang X, Hao J et al. Contribution of the organic anion transporter OAT2 to the renal active tubular secretion of creatinine and mechanism for serum creatinine elevations caused by cobicistat. Kidney Int. 86, 350-357 (2014).

21. German P, Szarcberg J, Hepner M et al. Effect of cobicistat on glomerular filtration rate in subjects with normal and impaired renal function. J. Acquir. Immune Defic. Syndr. 61(1), 32-40 (2012).

22. Horberg M, Tang B, Towner W et al. Impact of tenofovir on renal function in HIV-infected, antiretroviral-naïve patients. J. Acquir. Immune Defic. Syndr. 53(1), 62-69 (2010).

23. Bedimo R, Maalouf NM, Zhang $\mathrm{S}$ et al. Osteoporotic fracture risk associated with cumulative exposure to tenofovir and other antiretroviral agents. AIDS 26, 825-831 (2012).

24. Ruane PJ, DeJesus E, Berger D et al. Antiviral activity, safety, and pharmacokinetics/pharmacodynamics of tenofovir alafenamide as 10-day monotherapy in HIV-1 positive adults. J. Acquir. Immune Defic. Syndr. 63(4), 449-455 (2013).

25. Sax PE, Zolopa A, Brar I et al. Tenofovir alafenamide vs tenofovir disoproxil fumerate in single tablet regimens for initial HIV-1 therapy: a randomized Phase II study. J. Acquir. Immune Defic. Syndr 67(1), $52-58$ (2014).

26. Sax PE, Wohl D, Yin MT et al. Tenofovir alafenamide versus tenofovir disoproxil fumerate, coformulated with elvitegravir, cobicistat, and emtricitabine, for initial treatment of HIV-1 infection: two randomized, double-blind, Phase III, non-inferiority trials. Lancet 385, 2606-2615 (2015).

27. Pozniak A, Flamm J, Antinori A et al. Switching to the single-tablet regimen of elvitegravir, cobicistat, emtricitabine, and tenofovir DF from non-nucleoside reverse transcriptase inhibitor plus coformulated emtricitabine and tenofovir DF regimens: week 96 results of STRATEGY-NNRTI. HIV Clin. Trials 18(4), 141-148 (2017).

28. Arribas JR, DeJesus E, van Lunzen J et al. Simplification to single-tablet regimen of elvitegravir, cobicistat, emtricitabine, tenofovir DF from multi-tablet ritonavir-boosted protease inhibitor plus coformulated emtricitabine and tenofovir DF regimens: week 96 results of STRATEGY-PI. HIV Clin. Trials 18(3), 118-125 (2017).

29. Huhn GD, Tebas P, Gallant J et al. A randomized, open-label trial to evaluate switching to elvitegravir/cobicistat/emtricitabine/tenofovir alafenamide plus darunavir in treatment-experienced HIV-1 infected adults. J. Acquir. Immune Defic. Syndr. 74(2), 193-200 (2017).

- Important switch study in treatment-experienced patients that showed the benefit of a simplified two tablet once daily regimen was as good as maintaining current salvage regimen, but with better patient satisfaction.

30. Ross L, Parkin N, Chappey C et al. Phenotypic impact of HIV reverse transcriptase M184I/V mutations in combination with single thymidine analog mutations on nucleoside reverse transcriptase inhibitor resistance. AIDS 18, 1691-1696 (2004).

31. Perez-Valero I, Llibre JM, Lazzarin A et al. A Phase IIIb, open label, pilot study to evaluate switching to elvitegravir/cobicistat/emtricitabine/tenofovir alafenamide (EVG/COBI/FTC/TAF) in virologically-suppressed HIV-1 infected adult subjects harboring the NRTI resistance mutation M184V and /or M184I (GS-US-292-1824): week 24 results. Presented at: Program and Abstracts of the 22nd International AIDS Conference. Amsterdam, The Netherlands, 23-27 July 2018.

32. Genvoya US Prescribing Information. Gilead Sciences, Inc., CA, USA, 94404. www.gilead.com/-/media/files/pdfs/medicines/hiv/genvoya/genvoya_pi.pdf

33. Walmsley SL, Antela A, Clumeck N et al. Dolutegravir plus abacavir-lamivudine for the treatment of HIV-1 infection. N. Engl. J. Med. 369(19), 1807-1818 (2013).

34. Ford N, Shubber Z, Pozniak A et al. Comparative safety and neuropsychiatric adverse events associated with efavirenz use in first-line antiretroviral therapy: a systematic review and meta-analysis of randomized trials. J. Acquir. Immune Defic. Syndr. 69(4), 422-429 (2015).

35. Walmsley S, Baumgarten A, Berenguer J et al. Dolutegravir plus abacavir/lamivudine for the treatment of HIV-1 infection in antiretroviral therapy-naïve patients: week 96 and 144 results from the SINGLE randomized clinical trial. J. Acquir. Immune Defic. Syndr. 70(5), 515-519 (2015).

- SINGLE study was first study to show integrase strand transfer inhibitor (dolutegravir) was superior to Atripla (gold standard at the time) for HIV-1 treatment naive adults.

36. Raffi F, Jaeger H, Quiros-Roldan E et al. Once -daily dolutegravir versus twice daily raltegravir in antiretroviral-naïve adults with HIV-1 infection (SPRING-2 study): 96 week results from a randomized, double-blind, non-inferiority trial. Lancet Infect Dis. 11, 927-935 (2013).

37. Reese MJ, Savina PM, Generaux GT et al. In vitro investigations into the roles of drug transporters and metabolizing enzymes in the disposition and drug interactions of dolutegravir, a HIV integrase inhibitor. Drug Metab. Dispos. 41, 353-361 (2013).

38. Molina JM, Clotet B, van Lunzen J et al. Once daily dolutegravir versus darunavir plus ritonavir for treatment-naïve adults with HIV-1 infection (FLAMINGO): 96 week results from a randomized, open-label, Phase IIIb study. Lancet HIV 4, e127-e136 (2015). 
39. Cahn P, Pozniak AL, Mingrone $\mathrm{H}$ et al. Dolutegravir versus raltegravir in antiretroviral-experienced, integrase-inhibitor-naïve adults with HIV: week 96 results from the randomized, double-blind, non-inferiority SAILING study. Lancet 382(9893), 700-708 (2013).

40. Castagna A, Maggiolo F, Penco G et al. Dolutegravir in antiretroviral-experienced patients with raltegravir and/or elvitegravir resistant HIV-1: 24-week results of the Phase III VIKING-3 study. J. Inf. Dis. 210(3), 354-362 (2014).

41. Orell C. DoLPHIN-1: DTG or EFV+NRTIs in pregnant women initiating ART during third trimester. Presented at: Programs and abstracts of the 22nd International AIDS Conference. Amsterdam, The Netherlands, 23-27 July 2018.

42. Stek AM, Mirochnick M, Capparelli E et al. Reduced lopinavir exposure during pregnancy. AIDS 20, 1931-1939 (2006).

43. Mulligan N, Best BM, Wang J et al. Dolutegravir pharmacokinetics in pregnant and postpartum women living with HIV. AIDS 32, 729-737 (2018).

44. Zash R, Makhema J, Shapiro RL. Neural tube defects with dolutegravir treatment from the time of conception. N. Engl. J. Med. 379(10), 979-981 (2018).

45. U.S. Food and Drug Administration (FDA). Drug Safety Communication. HIV/AIDS Bureau, MD, USA, 20857 (2018). www.fda.gov/Drugs/DrugSafety/ucm608112.htm

46. Zash. Tsepamo: DTG exposure at conception associated with smaller increase in incidence of neural tube defects in updated analysis. Presented at: 10th IAS Conference on HIV Science. Mexico City, Mexico, 21-24 July 2019.

47. Zash R, Holmes L, Diseko M, Jacobson DL, Brummel S et al. Neural tube defects and antiretroviral treatment regimens in Botswana. $N$. Engl. J. Med. doi:10.1056/NEJMoa1905230 (2019) (Epub ahead of print).

- Most recent updated neural tube defect data in women on ART in Botswana and the implications for dolutegravir going forward.

48. WHO. News release: WHO recommends dolutegravir as preferred HIV treatment option in all populations. Mexico City, Mexico (2019). www.who.int/news-room/detail/22-07-2019

49. Menard A, Meddeb L, Tissot-Dupont $\mathrm{H}$ et al. Dolutegravir and weight gain: an unexpected bothering side effect? AIDS 31(10), 1499-1502 (2017).

50. Norwood J, Turner M, Bofill C et al. Weight gain in persons with HIV switched from efavirenz-based to integrase strand transfer inhibitor-based regimens. J. Acquir. Immune Defic. Syndr. 76(5), 527-531 (2017).

51. Bourgi K, Rebeiro PF, Turner M et al. Greater weight gain in treatment naïve persons starting dolutegravir-based antiretroviral therapy. Clin. Infect. Dis. (2019) (Epub ahead of print).

52. de Boer M, van den Berk G, van Holten $\mathrm{N}$ et al. Intolerance of dolutegravir-containing combination antiretroviral therapy regimens in real-life clinical practice. AIDS 30(18), 2831-2834 (2016).

53. Elzi L, Erb S, Furrer H et al. Adverse events of raltegravir and dolutegravir. AIDS 31(13), 1853-1858 (2017).

54. Hoffman C, Llibre JM. Neuropsychiatric adverse events with dolutegravir and other integrase strand transfer inhibitors. AIDS Rev. 21(4), 4-10 (2019).

55. GlaxoSmithKline. Tivicay US Prescribing Information. GlaxoSmithKline, Research Triangle Park, NC 27709, USA. www.gsksource.com/pharma/content/dam/GlaxoSmithKline/US/en/Prescribing_Information/Tivicay/pdf/TIVICAY-PI-PIL.PDF

56. Tsiang M, Jones GS, Goldsmith J et al. Antiviral activity of bictegravir (GS-9883), a novel potent HIV-1 integrase strand transfer inhibitor with an improved resistance profile. Antimicrob. Agents Chemother. 60(12), 7086-7097 (2016).

57. Andreatta K, Willkom M, Martin R et al. Switching to bictegravir/emtricitabine/tenofovir alafenamide maintained HIV-1 RNA suppression in participants with archived antiretroviral resistance including M184V/I. J. Antimicrob.

Chemother. Doi:10.1093/jac/dkz347 (2019) (Epub ahead of print).

\section{- Showing that switching to Biktarvy in patients with history of $\mathrm{M184V} / \mathrm{I}$ mutation is reasonable.}

58. Daar ES, DeJesus E, Ruane P et al. Efficacy and safety of switching to fixed-dose bictegravir, emtricitabine, and tenofovir alafenamide from boosted protease inhibitor-based regimens in virologically suppressed adults with HIV-1: 48 week results of a randomised, open-label, multicenter, Phase III, non-inferiority trial. Lancet HIV. 5, 347-356 (2018).

59. Molina JM, Ward D, Brar I et al. Switching to fixed-dose bictegravir, emtricitabine, and tenofovir alafenamide from dolutegravir plus abacavir and lamivudine in virologically suppressed adults with HIV-1: 48 week results of a randomised, double-blind, multicentre, active-controlled, Phase III, non-inferiority trial. Lancet HIV. 5, 357-365 (2018).

60. Wohl DA, Yazdanpanah Y, Baumgarten A et al. Bictegravir combined with emtricitabine and tenofovir alafenamide versus dolutegravir, abacavir, and lamivudine for initial treatment of HIV-1 infection: week 96 results from a randomized, double-blind, multicenter, Phase III, non-inferiority trial. Lancet HIV. 6(6), e355-e363 (2019).

- Head-to-head comparison study between Biktarvy and Triumeq, the two most important single tablet integrase strand transfer inhibitor regimens showing equivalence.

61. Stellbrink HJ, Arribas JR, Stephens JL et al. Co-formulated bictegravir, emtricitabine, and tenofovir alafenamide versus dolutegravir with emtricitabine and tenofovir alafenamide for initial treatment of HIV-1 infection: week 96 results from a randomized, double-blind, multicenter, Phase III, non-inferiority trial. Lancet HIV. 6(6), e364-e372 (2019). 
62. Biktarvy US Prescribing Information. Gilead Sciences, Inc, CA, USA, 94404. www.gilead.com/ /media/files/pdfs/medicines/hiv/biktarvy/biktarvy_pi.pdf

63. Wada N, Jacobson LP, Cohen M et al. Cause-specific mortality among HIV-infected individuals, by CD4 ${ }^{(+)}$cell count at HAART initiation, compared with HIV - uninfected individuals. AIDS 28(2), 257-265 (2014).

64. Spreen W, Ford SL, Chen S et al. GSK1265744 pharmacokinetics in plasma and tissue after single-dose long-acting injectable administration in healthy subjects. J. Acquir. Immune Defic. Syndr. 67(5), 481-486 (2014).

65. Spreen W, Williams P, Margolis D et al. Pharmacokinetics, safety, and tolerability with repeat doses of GSK1265744 and rilpivirine (TMC278) long-acting nanosuspensions in healthy adults. J. Acquir. Immune Defic. Syndr. 67(5), 487-492 (2014).

66. Margolis DA, Brinson CC, Smith GH et al. Cabotegravir plus rilpivirine, once a day, after induction with cabotegravir plus nucleoside reverse transcriptase inhibitors in antiretroviral naïve adults with HIV-1 infection (LATTE): a randomised, Phase IIb, dose-ranging trial. Lancet Infect. Dis. 15, 1145-1155 (2015).

67. Margolis DA, Gonzalez-Garcia J, Stellbrink HJ et al. Long-acting intramuscular cabotegravir and rilpivirine in adults with HIV-1 infection (LATTE-2): 96-week results of a randomised, open-label, Phase IIb, non-inferiority trial. Lancet 390(10101), 1499-1510 (2017).

- LATTE-2 study that sets the stage for proof of concept for long-acting cabotegravir + long-acting rilpivirine injection for maintenance therapy for treatment naive patients.

68. Swindells S, Andrade-Villanueva JF, Richmond GJ et al. Long acting cabotegravir+rilpivirine for maintenance therapy: ATLAS week 48 results. Presented at: Conference on Retroviruses and Opportunistic Infections. Seattle, WA, USA, 4-7 March 2019. (Abstract 1475).

69. Murry. ATLAS: patient-reported outcomes from Phase III study of switch to long-acting injectable cabotegravir+rilpivirine in virologically suppressed patients. Presented at: 10th IAS Conference on HIV Science. Mexico City, Mexico, 21-24 July 2019. (Abstract MOAB0103).

70. Orkin C, Arasteh K, Hernandez-More MG et al. Long-acting cabotegravir+rilpivirine for HIV maintenance: FLAIR week 48 results. Presented at: Conference on Retroviruses and Opportunistic Infections. Seattle, WA, USA, 4-7 March 2019. (Abstract 3947).

71. Grant RM, Lama JR, Anderson PL et al. Preexposure chemoprophylaxis for HIV prevention in men who have sex with men. N. Engl. J. Med. 363, 2587-2599 (2010).

72. Thigpen MC, Kebaabetswe PM, Paxton LA et al. Antiretroviral preexposure prophylaxis for heterosexual HIV transmission in Botswana. N. Eng. J. Med. 367, 423-434 (2012).

73. Markowitz M, Frank I, Grant RM et al. Safety and tolerability of long-acting cabotegravir injection in HIV-uninfected men (ÉCLAIR): a multicenter, double-blind, randomised, placebo-controlled, Phase IIa trial. Lancet HIV. 4, 331-340 (2017).

- ECLAIR trial for cabotegravir for pre-exposure prophylaxis showing that the pharmacokinetic analysis led to a change in the dosing interval for long-acting cabotegravir for pre-exposure prophylaxis.

74. Landovitz RJ, Li S, Grinsztejn B et al. Safety, tolerability, and pharmacokinetics of long-acting injectable cabotegravir in low-risk HIV-uninfected individuals: HPTN 077, a Phase IIa randomized controlled trial. PLoS Med. 15(11), 1-22 (2018).

- HPTN 077 showed that the revised dosing schedule for long-acting cabotegravir was effective and achieved plasma levels, considered protective for preventing HIV infection.

75. Press Release. ATLAS-2M study met its primary endpoint, showing similar efficacy of cabotegravir and rilpivirine administered every eight weeks compared to four-week administration. (2019). http://clinicaltrials.gov/ct2/show/NCT03299049

76. Raffi F, Babiker AG, Richert I et al. Ritonavir-boosted darunavir combined with raltegravir or tenofovir-emtricitabine in antiretroviral-naïve adults infected with HIV-1: 96 week results from the NEAT001/ANRS143 randomised non-inferiority trial. Lancet 384, 1942-1951 (2014).

77. Riddler SA, Haubrich R, DiRienzo AG et al. Class-sparing regimens for initial treatment of HIV-1 infection. N. Engl. J. Med. 358, 2095-2106 (2008).

78. Llibre JM, Hung CC, Brinson C et al. Efficacy, safety, and tolerability of dolutegravir-rilpivirine for the maintenance of virological suppression in adults with HIV-1: Phase III, randomised, non-inferiority SWORD-1 and SWORD-2 studies. Lancet 391(10123), 839-849 (2018).

- SWORD-1 and 2 studies that showed two-drug therapy was effective and safe for patients virologically suppressed on three-drug ART who want to switch to two-drug therapy.

79. Cahn P, Sierra-Madero J, Arribas JR et al. Dolutegravir plus lamivudine versus dolutegravir plus tenofovir disoproxil fumerate and emtricitabine in antiretroviral-naïve adults with HIV-1 infection (GEMINI-1 and GEMINI-2): week 48 results from two multicenter, double-blind, randomised, non-inferiority, Phase III trials. Lancet 393(10167), 143-155 (2019).

- GEMINI 1 and 2 show that two-drug therapy with dolutegravir + lamivudine is noninferior to traditional three-drug regimen recommended by the Department of Health and Human Services treatment guidelines.

80. Cahn. GEMINI-1 and -2: DTG + 3TC noninferior to DTG + FTC/TDF at Wk 96 in ART-naïve adults. Presented at: 10th IAS Conference on HIV Science. Mexico City, Mexico, 21-24 July 2019. (Abstract WEAB0404LB). 
81. Canducci F, Marinozzi MC, Sampaolo M et al. Genotypic/phenotypic patterns of HIV-1 integrase resistance to raltegravir. J. Antimicrob. Chemother. 65, 425-433 (2010).

82. Sichtig N, Sierra S, Kaiser R et al. Evolution of raltegravir resistance during therapy. J. Antimicrob. Chemother. 64, 25-32 (2009).

83. $\mathrm{Hu} \mathrm{Z}$, Kuritzkes DR. Effect of raltegravir resistance mutations in HIV-1 integrase on viral fitness. J. Acquir. Immune Defic. Syndr. 55(2), $148-155$ (2010).

84. Abram ME, Hluhanich RM, Goodman DD et al. Impact of primary elvitegravir resistance-associated mutations in HIV-1 integrase on drug susceptibility and viral replication fitness. Antimicrob. Agents Chemother. 57(6), 2654-2663 (2013).

85. Hare S, Smith SJ, Metifiot $M$ et al. Structural and functional analyses of the second-generation integrase strand transfer inhibitor dolutegravir (S/GSK1349572). Mol. Pharmacol. 80(4), 565-572 (2011).

86. Rhee SY, Grant PM, Tzou PL et al. A systematic review of the genetic mechanisms of dolutegravir resistance. J. Antimicrob. Chemother. (2019) (Epub ahead of print).

87. Hocqueloux L, Raffi F, Prazuck T et al. Dolutegravir monotherapy versus dolutegravir/abacavir/lamivudine for virologically suppressed people living with chronic HIV infection: the randomized non-inferiority MONCAY trial. Clin. Infect. Dis. (2019) (Epub ahead of print).

88. Tsiang M, Jones GS, Goldsmith J et al. Antiviral activity of bictegravir (GS-9883), a novel potent HIV-1 integrase strand transfer inhibitor with an improved resistance profile. Antimicrob. Agents Chemother. 60(12), 7086-7097 (2016).

89. Smith SJ, Zhao XZ, Burke TR, Hughes SH. Efficacies of cabotegravir and bictegravir against drug-resistant HIV-1 integrase mutants. Retrovirology 15(1), 37 (2018).

90. Oliveira M, Ibanescu RI, Anstett $\mathrm{K}$ et al. Selective resistance profiles emerging in patient-derived clinical isolates with cabotegravir, bictegravir, dolutegravir, and elvitegravir. Retrovirology 15(1), 56 (2018).

91. Karmon SL, Mohri H, Spreen W, Markowitz M. GSK1265744 demonstrates robust in vitro activity against various clades of HIV-1. J. Acquir. Immune Defic. Syndr. 68(3), e39-e41 (2015).

92. Koullias Y, Sax PE, Fields NF et al. Should we be testing for baseline integrase resistance in patients newly diagnosed with human immunodeficiency virus? Clin. Infect. Dis. 65, 1274-1281 (2017).

93. McGee KS, Okeke NL, Hurt CB, McKellar MS. Canary in the coal mine? Transmitted mutations conferring resistance to all integrase strand transfer inhibitors in a treatment-naïve patient. Open Forum Infect. Dis. 5(11), ofy294 (2018). 
